\title{
Making and breaking the exciton in layered halide hybrid perovskites
}

\author{
M. Kepenekian ${ }^{1}$, B. Traore, J.-C. Blancon ${ }^{2}$, H. Tsai ${ }^{2}$, W. Nie ${ }^{2}$, C. Stoumpos ${ }^{3}$, L. Pedesseau ${ }^{4}$, \\ C. Katan $^{1}$, S. Tretiak ${ }^{2}$, M. G. Kanatzidis ${ }^{3}$, J. Even ${ }^{4}$ and A. D. Mohite ${ }^{2}$ \\ ${ }^{1}$ Institut des Sciences Chimiques de Rennes (ISCR), CNRS, Rennes, France. \\ ${ }^{2}$ Los Alamos National Laboratory, Los Alamos, New Mexico, USA. \\ ${ }^{3}$ Department of Chemistry, Northwestern University, Evanston, Illinois, USA. \\ ${ }^{4}$ FOTON, CNRS, INSA Rennes, Rennes, France. \\ mikael.kepenekian@univ-rennes1.fr
}

Layered halide hybrid organic-inorganic perovskites [1] have been the subject of intense investigation before the rise of three-dimensional (3D) halide perovskites and their impressive performance in solar cells. Recently, layered perovskites have also been proposed as attractive alternatives for photostable solar cells [2] and revisited for light-emitting devices. Interestingly, these performances can be traced back to extremely efficient internal exciton dissociation through edge states identified on thin films and single crystals [3].

Layered perovskites present fascinating features with inherent quantum and dielectric confinements imposed by the organic layers sandwiching the inorganic core, and computational approaches have successfully help rationalized their properties (excitonic, Rashba effects, etc.) [4-6]. Here, we propose a joint spectroscopic and computational investigation to unravel the origin of the recently identified layer-edge states in layered Ruddlesden-Popper phases with inorganic layers containing $n=1$ to 4 octahedra. We show that for $n>2$, the system presents a localized surface state within the band gap.

Based on our conclusion, we propose an elastic model providing design principles for future layered perovskites with optimized properties for photovoltaics or light emission.

\section{References}

[1] L. Pedesseau et al., ACS Nano (2016), 10, 9776.

[2] H. Tsai et al., Nature (2016), 536, 312.

[3] J.-C. Blancon et al., Science (2017), 355, 1288.

[4] M. Kepenekian et al., ACS Nano (2015), 12, 11557.

[5] D. Sapori, M. Kepenekian, L. Pedesseau, C. Katan, J. Even, Nanoscale (2016), 8, 6369.

[6] M. D. Smith et al., Chem. Sci. (2017), 8, 1960. 